Also available at http://amc-journal.eu

ISSN 1855-3966 (printed edn.), ISSN 1855-3974 (electronic edn.)

ARS MATHEMATICA CONTEMPORANEA 9 (2015) 109-113

\title{
On spectral radius and energy of complete multipartite graphs
}

\author{
Dragan Stevanović * \\ University of Primorska, UP IAM, Muzejski trg 2, SI-6000 Koper, Slovenia and \\ Mathematical Institute, Serbian Academy of Science and Arts, Knez Mihajlova 36, 11000 \\ Belgrade, Serbia \\ Ivan Gutman \\ Faculty of Science, University of Kragujevac, P.O. Box 60, 34000 Kragujevac, Serbia \\ Masood U. Rehman \\ Air University, Islamabad, Pakistan
}

Received 18 June 2013, accepted 8 March 2014, published online 8 August 2014

\begin{abstract}
Let $K_{n_{1}, n_{2}, \ldots, n_{p}}$ denote the complete $p$-partite graph, $p>1$, on $n=n_{1}+n_{2}+\cdots+n_{p}$ vertices and let $n_{1} \geq n_{2} \geq \cdots \geq n_{p}>0$. We show that for a fixed value of $n$, both the spectral radius and the energy of complete $p$-partite graphs are minimal for complete split graph $C S(n, p-1)$ and are maximal for Turán graph $T(n, p)$.

Keywords: Spectral radius of graph, graph energy, complete multipartite graph, complete split graph, Turán graph.

Math. Subj. Class.: 05C50
\end{abstract}

\section{Introduction}

Let $G$ be a simple graph on $n$ vertices, and $\lambda_{1} \geq \lambda_{2} \geq \cdots \geq \lambda_{n}$ be its eigenvalues (i.e., the eigenvalues of the $(0,1)$-adjacency matrix of $G)$ [2, 4]. Then $\lambda_{1}=\lambda_{1}(G)$ is said to be the spectral radius of the graph $G$ whereas

$$
\mathcal{E}=\mathcal{E}(G)=\sum_{i=1}^{n}\left|\lambda_{i}\right|
$$

* Corresponding author. The work of DS was supported by Research Programme P1-0285 and Research Project J1-4021 of the Slovenian Research Agency and Research Grant ON174033 of the Ministry of Education and Science of Serbia.

E-mail addresses: dragan.stevanovic@upr.si (Dragan Stevanović), gutman@kg.ac.rs (Ivan Gutman), (Masood U. Rehman)

(a) (i) This work is licensed under http://creativecommons.org/licenses/by/3.0/ 
is its energy. The spectral radius is one of the most thoroughly investigated graph-spectral parameters [2, 4, 3]. Also the graph energy has recently attracted much attention [6, 7]. In spite of this, not much is known on $\lambda_{1}$ and $\mathcal{E}$ of complete multipartite graphs [5].

Let $p>1$. Denote by $K_{n_{1}, n_{2}, \ldots, n_{p}}$ the complete $p$-partite graph on $n=n_{1}+n_{2}+\cdots+$ $n_{p}$ vertices. For convenience, we assume that $n_{1} \geq n_{2} \geq \cdots \geq n_{p}>0$. Two particular types of complete multipartite graphs are:

- Complete split graph $C S(n, p-1)=K_{n-p+1,1, \ldots, 1}$ consisting of an independent set of $n-p+1$ vertices and a clique of $p-1$ vertices, such that each vertex of the independent set is adjacent to each vertex of the clique, and

- Turán graph $T(n, p) \cong K_{\lceil n / p\rceil, \ldots,\lceil n / p\rceil,\lfloor n / p\rfloor, \ldots,\lfloor n / p\rfloor}$, the $(p+1)$-clique-free graph with maximum number of edges [10].

A fundamental result on the spectrum of complete multipartite graphs is:

Theorem 1.1 ([9, 8]). A connected graph has exactly one positive eigenvalue of its adjacency matrix if and only if it is a complete multipartite graph.

An immediate consequence of Theorem 1.1 is

Lemma 1.2. If $\lambda_{1}$ is the spectral radius of the complete multipartite graph $K_{n_{1}, n_{2}, \ldots, n_{p}}$, then $\mathcal{E}\left(K_{n_{1}, n_{2}, \ldots, n_{p}}\right)=2 \lambda_{1}$.

Proof. Since all graph eigenvalues are real numbers and their sum is zero, from Eq. (1.1) follows that $\mathcal{E}(G)$ is equal to twice the sum of positive eigenvalues.

It is known that the characteristic polynomial of $K_{n_{1}, n_{2}, \ldots, n_{p}}$ is given by [2, 5]

$$
\phi\left(K_{n_{1}, n_{2}, \ldots, n_{p}}, \lambda\right)=\lambda^{n-p}\left(1-\sum_{i=1}^{p} \frac{n_{i}}{\lambda+n_{i}}\right) \prod_{j=1}^{p}\left(\lambda+n_{j}\right) .
$$

The spectrum of $K_{n_{1}, n_{2}, \ldots, n_{p}}$ consists of the spectral radius $\lambda_{1}$ determined from the equation $\sum_{i=1}^{p} \frac{n_{i}}{\lambda+n_{i}}=1$, eigenvalue 0 with multiplicity $n-p$ and $p-1$ eigenvalues situated in the intervals $\left[-n_{p},-n_{p-1}\right], \ldots,\left[-n_{2},-n_{1}\right]$. In the special case $n_{1}=n_{2}=\cdots=n_{p}=t$, the spectrum of $K_{t, t, \ldots, t}$ consists of the spectral radius $t(p-1)$ with unit multiplicity, eigenvalue 0 with multiplicity $p(t-1)$, and eigenvalue $-t$ with multiplicity $p-1$, so that

$$
\mathcal{E}\left(K_{t, t, \ldots, t}\right)=2(p-1) t .
$$

Remark 1.3. The 1-partite complete graph (when $p=1$ and $t=n$ ) is the edgeless graph $\overline{K_{n}}$ for which, consistently, $\lambda_{1}=\mathcal{E}=0$. The $n$-partite complete graph (when $p=n$ and $t=1$ ) is the ordinary complete graph $K_{n}$ for which, consistently, $\lambda_{1}=n-1$ and $\mathcal{E}=2(n-1)$.

If $p=2$, then the special case of Eq. (1.2) is

$$
\phi\left(K_{n_{1}, n_{2}}, \lambda\right)=\lambda^{n-2}\left(\lambda^{2}-n_{1} n_{2}\right),
$$

from which the (well known) expressions for spectral radius and energy follow:

$$
\lambda_{1}\left(K_{n_{1}, n_{2}}\right)=\sqrt{n_{1} n_{2}}, \quad \mathcal{E}\left(K_{n_{1}, n_{2}}\right)=2 \sqrt{n_{1} n_{2}} .
$$

Because $n_{1}+n_{2}=n$, for a fixed number of vertices $n$, we arrive at: 
Claim 1.4. $1^{\circ} \lambda_{1}\left(K_{n_{1}, n_{2}}\right)$ and $\mathcal{E}\left(K_{n_{1}, n_{2}}\right)$ are minimal if $n_{1}=n-1$ and $n_{2}=1$. $2^{\circ} \lambda_{1}\left(K_{n_{1}, n_{2}}\right)$ and $\mathcal{E}\left(K_{n_{1}, n_{2}}\right)$ are maximal if $n_{1}-n_{2} \leq 1$.

If $p=3$, then the special case of Eq. (1.2) is

$$
\phi\left(K_{t_{1}, t_{2}, t_{3}}, \lambda\right)=\lambda^{p-3}\left(\lambda^{3}-\left(t_{1} t_{2}+t_{2} t_{3}+t_{3} t_{1}\right) \lambda-2 t_{1} t_{2} t_{3}\right) .
$$

Using (1.3), it is relatively easy to show the following:

Claim 1.5. $1^{\circ}$ Let $n_{1}+n_{2}+n_{3}$ be equal to a fixed integer $n$. Then the spectral radius and the energy of $K_{n_{1}, n_{2}, n_{3}}$ are minimal if $n_{1}=n-2$ and $n_{2}=n_{3}=1$.

$2^{\circ}$ Let $n_{1}+n_{2}+n_{3}$ be equal to a fixed integer $n$. Then the spectral radius and the energy of $K_{n_{1}, n_{2}, n_{3}}$ are maximal if $n_{1}-n_{3} \leq 1$.

The above claims were the motivation for establishing our main results:

Theorem 1.6. Let $p \geq 2$ and $n_{1}+n_{2}+\cdots+n_{p}$ be equal to a fixed integer $n$. Then the spectral radius and the energy of $K_{n_{1}, n_{2}, \ldots, n_{p}}$ are minimal if $K_{n_{1}, n_{2}, \ldots, n_{p}} \cong C S(n, p-1)$.

Theorem 1.7. Let $p \geq 2$ and $n_{1}+n_{2}+\cdots+n_{p}$ be equal to a fixed integer $n$. Then the spectral radius and the energy of $K_{n_{1}, n_{2}, \ldots, n_{p}}$ are maximal if $K_{n_{1}, n_{2}, \ldots, n_{p}} \cong T(n, p)$.

\section{Proofs of Theorems $\mathbf{1 . 6}$ and $\mathbf{1 . 7}$}

Let $\lambda_{1}$ and $x$ be, respectively, the spectral radius and the corresponding unit eigenvector of the adjacency matrix of $K_{n_{1}, \ldots, n_{p}}$. Since $\lambda_{1}$ is a simple eigenvalue of $K_{n_{1}, \ldots, n_{p}}$, similar vertices have equal $x$-components. Hence, we may denote by $x_{i}$ the common $x$-component of vertices in the part of $K_{n_{1}, \ldots, n_{p}}$ having $n_{i}$ vertices for $i=1, \ldots, p$. From the eigenvalue equation, we have:

$$
\lambda_{1} x_{i}=\sum_{\substack{k=1 \\ k \neq i}}^{p} n_{k} x_{k}=X-n_{i} x_{i}
$$

where $X=\sum_{k=1}^{p} n_{k} x_{k}$. Then

$$
x_{i}=\frac{X}{\lambda_{1}+n_{i}} .
$$

Lemma 2.1. If $n_{i}-n_{j} \geq 2$, then

$$
\lambda_{1}\left(K_{n_{1}, \ldots, n_{i}-1, \ldots, n_{j}+1, \ldots, n_{p}}\right)>\lambda_{1}\left(K_{n_{1}, \ldots, n_{i}, \ldots, n_{j}, \ldots, n_{p}}\right) .
$$

Proof. Let $\lambda_{1}, x$ and $E$ denote, respectively, the spectral radius, the corresponding eigenvector, and the edge set of $K_{n_{1}, \ldots, n_{i}, \ldots, n_{j}, \ldots, n_{p}}$, and let $\lambda_{1}^{*}, A^{*}$ and $E^{*}$ denote, respectively, the spectral radius, the adjacency matrix, and the edge set of $K_{n_{1}, \ldots, n_{i}-1, \ldots, n_{j}+1, \ldots, n_{p}}$. From the Variational theorem we have

$$
\begin{aligned}
\lambda_{1}^{*} \geq x^{T} A^{*} x & =\sum_{u v \in E^{*}} 2 x_{u} x_{v} \\
& =\sum_{u v \in E} 2 x_{u} x_{v}+\sum_{u v \in E^{*} \backslash E} 2 x_{u} x_{v}-\sum_{u v \in E \backslash E^{*}} 2 x_{u} x_{v} \\
& =\lambda_{1}+2 x_{i}\left(n_{i}-1\right) x_{i}-2 x_{i} n_{j} x_{j} \\
& =\lambda_{1}+2 x_{i} X\left(\frac{n_{i}-1}{\lambda_{1}+n_{i}}-\frac{n_{j}}{\lambda_{1}+n_{j}}\right)
\end{aligned}
$$


by Eq. (2.1). Next, note that $K_{n_{1}, \ldots, n_{i}, \ldots, n_{j}, \ldots, n_{p}}$ has $K_{n_{i}, n_{j}}$ as an induced subgraph, so that, by the Interlacing theorem [2],

$$
\lambda_{1} \geq \sqrt{n_{i} n_{j}}>n_{j} .
$$

Therefore,

$$
\frac{n_{i}-1}{\lambda_{1}+n_{i}}-\frac{n_{j}}{\lambda_{1}+n_{j}}=\frac{\left(n_{i}-n_{j}-1\right) \lambda_{1}-n_{j}}{\left(\lambda_{1}+n_{i}\right)\left(\lambda_{1}+n_{j}\right)} \geq \frac{\lambda_{1}-n_{j}}{\left(\lambda_{1}+n_{i}\right)\left(\lambda_{1}+n_{j}\right)}>0
$$

so that $\lambda_{1}^{*}>\lambda_{1}$ follows from Eq. (2.2).

Proof of Theorem 1.6 Let $K_{m_{1}, \ldots, m_{p}}$ be the complete multipartite graph with the smallest spectral radius. If there are two parameters $m_{i} \geq m_{j} \geq 2$, then $\left(m_{i}+1\right)-\left(m_{j}-1\right) \geq 2$ and from Lemma 2.1

$$
\lambda_{1}\left(K_{m_{1}, \ldots, m_{i}, \ldots, m_{j}, \ldots, m_{p}}\right)>\lambda_{1}\left(K_{m_{1}, \ldots, m_{i}+1, \ldots, m_{j}-1, \ldots, m_{p}}\right)
$$

contradicting the choice of $K_{m_{1}, \ldots, m_{i}, \ldots, m_{j}, \ldots, m_{p}}$. Hence, all parameters $m_{1}, \ldots, m_{p}$ are equal to one, except for one parameter equal to $n-p+1$, so that $K_{m_{1}, \ldots, m_{p}} \cong C S(n, p-1)$.

Proof of Theorem 1.7 Let $K_{m_{1}, \ldots, m_{p}}$ be the complete multipartite graph with the largest spectral radius. It is apparent from Lemma 2.1, that $\left|m_{i}-m_{j}\right| \leq 1$ holds for all $i \neq j$, as otherwise, assuming $m_{i}-m_{j} \geq 2$,

$$
\lambda_{1}\left(K_{m_{1}, \ldots, m_{i}-1, \ldots, m_{j}+1, \ldots, m_{p}}\right)>\lambda_{1}\left(K_{m_{1}, \ldots, m_{i}, \ldots, m_{j}, \ldots, m_{p}}\right)
$$

contradicting the choice of $K_{m_{1}, \ldots, m_{i}, \ldots, m_{j}, \ldots, m_{p}}$. The condition $\left|m_{i}-m_{j}\right| \leq 1$ for $i \neq j$ implies that each parameter $m_{i}$ is equal to either $\lfloor n / p\rfloor$ or $\lceil n / p\rceil$, so that $K_{m_{1}, \ldots, m_{p}} \cong$ $T(n, p)$.

Remark 2.2. Delorme [5] proved (a bit too concisely) that changing the arbitrary $e$ parameters of a complete multipartite graph by their average value increases the spectral radius by relying on the characteristic polynomial. While this result can be substituted for Lemma 2.1 in the proofs of Theorems 1.6 and 1.7, Lemma 2.1 is an independent result based on the principal eigenvector and inspired by the rotation lemma from [1].

Remark 2.3. Delorme also asked in [5] whether the spectral radius of complete multipartite graph $K_{n_{1}, \ldots, n_{p}}$ is a concave function on the $(p-1)$-dimensional simplex $Y$ : $\sum_{i=1}^{p} n_{i}=n \wedge(\forall i \in\{1, \ldots, p\})\left(n_{i} \geq 0\right)$, i.e., whether

$$
\lambda_{1}\left(K_{t\left(n_{1}, \ldots, n_{p}\right)+(1-t)\left(m_{1}, \ldots, m_{p}\right)}\right) \geq t \lambda_{1}\left(K_{n_{1}, \ldots, n_{p}}\right)+(1-t) \lambda_{1}\left(K_{m_{1}, \ldots, m_{p}}\right)
$$

for any two points $\left(n_{1}, \ldots, n_{p}\right),\left(m_{1}, \ldots, m_{p}\right) \in Y$ and each $t \in[0,1]$ such that

$$
t\left(n_{1}, \ldots, n_{p}\right)+(1-t)\left(m_{1}, \ldots, m_{p}\right) \in Y ?
$$

Delorme proved this affirmatively for $p \leq 3$ in [5]. We tested it computationally for $t=$ $0.5, p \in\{4, \ldots, 10\}$ and $n \leq 33$ and found no counterexamples to the above question on these simplices. 


\section{References}

[1] F. Belardo, E. M. Li Marzi, S. K. Simić, Some results on the index of unicyclic graphs, Linear Algebra Appl. 416 (2006), 1048-1059.

[2] D. Cvetković, M. Doob, H. Sachs, Spectra of Graphs-Theory and Application, Academic Press, New York, 1980.

[3] D. Cvetković, P. Rowlinson, The largest eigenvalue of a graph: A survey, Lin. Multilin. Algebra 28 (1990), 3-33.

[4] D. Cvetković, P. Rowlinson, S. Simić, An Introduction to the Theory of Graph Spectra, Cambridge Univ. Press, Cambridge, 2010.

[5] C. Delorme, Eigenvalues of complete multipartite graphs, Discrete Math. 312 (2012), 25322535.

[6] I. Gutman, The energy of a graph: Old and new results, in: A. Betten, A. Kohnert, R. Laue, A. Wassermann (eds.), Algebraic Combinatorics and Applications, Springer, Berlin, 2001, 196211.

[7] X. Li, Y. Shi, I. Gutman, Graph Energy, Springer, New York, 2012.

[8] M. M. Petrović, The spectrum of infinite complete multipartite graphs, Publ. Inst. Math. (Belgrade) (N.S.) 31(45) (1982), 169-176.

[9] J. H. Smith, Some properties of the spectrum of a graph, in: R. Guy, H. Hanani, N. Sauer, J. Schönheim (eds.), Combinatorial Structures and Their Applications, Gordon \& Breach, New York, 1970, 403-406.

[10] P. Turán, On an extremal problem in graph theory, Mat. Fiz. Lap. 48 (1941), 436-452. 